\title{
CHARACTERIZATION OF SERIES RESISTANCES AND MOBILITY ATTENUATION PHENOMENA IN SHORT CHANNEL MOS TRANSISTORS
}

\author{
A. EL ABBASSI ${ }^{a}, Y$. AMHOUCHE ${ }^{a}$, E. BENDADA $^{\text {b }}$, \\ R. RMAILY ${ }^{\mathrm{a}}$ and K. RAÏS ${ }^{\mathrm{a}, *}$ \\ ${ }^{a}$ Laboratoire de Caractérisation des Composants à Semi-conducteurs, \\ Université Chouaib, Doukkali B.P. 20, EL Jadida-Maroc; \\ ${ }^{\mathrm{b}}$ Laboratoire de la Microelectronique et de l'instrumentation, \\ Faculté des Sciences et Techniques, B.P. 509 Errachidia - Maroc
}

(Received 5 February 2001; In final form 19 March 2001)

\begin{abstract}
As the scaling proceeds, the transverse electric field increase, and a mobility attenuation phenomenon becomes of primordial interest. Indeed, the high transverse electric field is generally ascribed [1] to surface roughness scattering in the channel and consequently reduce effective mobility of carriers. In short channel MOSFET, the Source-Drain resistance $\mathbf{R}_{\mathbf{s d}}$ influence strongly the effective mobility.

The aim of this work is therefore to propose an original method especially conceived for the extraction of the series resistance $\mathbf{R}_{\text {sd }}$. Using the approach of the Surface Roughness Scattering which enables a consistent modelling of the mobility to be obtained from low to high electric field [2], this allow to determine all model parameters in particular a Series Resistance from the plot of the transfer characteristic drain current $I_{d}$ versus gate voltage $V_{g}$ Curves.
\end{abstract}

Keywords: MOS Transistor; Surface roughness; Effective mobility; Series resistance

\section{INTRODUCTION}

According to the results obtained by the chemical methods [1], the mobility decreases when the surface roughness on the MOSFET channel increases. The $\langle\langle$ periodicity $\rangle\rangle$ breach at the level of $\mathrm{Si} / \mathrm{SiO}_{2}$

*Corresponding author. 
interface leads composition undulations intermediate between $\mathrm{Si}$ and $\mathrm{SiO}_{2}$ of some Angstroms of height and some hundreds Angstroms of period [2]. This interface $\mathrm{Si} / \mathrm{SiO}_{2}$ structure introduce a surface roughness, which provokes the decrease of mobility. In this paper we propose a new method for determining the source-drain resistance $\mathbf{R}_{\text {sd }}$ which has the practical advantage first of avoiding problems due to the use of several channel length devices and second of being very sensitive to particular features of short channel MOSFETs.

\section{THEORY}

The model of variation mobility with effective field considers that the attenuation of effective mobility is particularly due to Surface Roughness Scattering [3]. When the transversal field increases, inversion charges are flattened on the weak mobility edge, on a length $\Delta$ of some Angströms near the interface $\mathrm{Si} / \mathrm{SiO}_{2}$. This work generalised all the classical models $[4,5]$, give a physical meaning to the different used parameters, examine the results of the chemical method [1] and allow to determine the series resistance.

The first order mobility low approximation at not too high electric field is given by [4]:

$$
\frac{1}{\mu_{\mathrm{eff}}}=\frac{1}{\mu_{0}} \cdot\left[1+\theta_{1}\left(V_{g}-V_{t}\right)\right]
$$

where $\mu_{0}$ is the low gate voltage mobility, $\theta_{1}$ is the mobility attenuation parameter, $\mathrm{V}_{\mathrm{g}}$ and $\mathrm{V}_{\mathrm{t}}$ are gate and threshold voltage respectively. This approach (1) doesn't describe correctly the devices with short channel length, because it does not explain the negative transconductance obtained experimentally in high electric field [2].

The other model of effective mobility described by [5] consists to introduce a second parameter attenuation $\theta_{2}$ :

$$
\frac{1}{\mu_{\mathrm{eff}}}=\frac{1}{\mu_{0}} \cdot\left[1+\theta_{1}\left(V_{g}-V_{t}\right)+\theta_{2}\left(V_{g}-V_{t}\right)^{2}\right]
$$

For the weak values of $\theta_{2}$, we refined the model [4]. Thought it explains the negative transconductance obtained for the strong value of $\mathrm{V}_{\mathrm{g}}$, (Fig. 1) but don't give a physical signification to $\theta_{1}$ and $\theta_{2}$ parameters. 


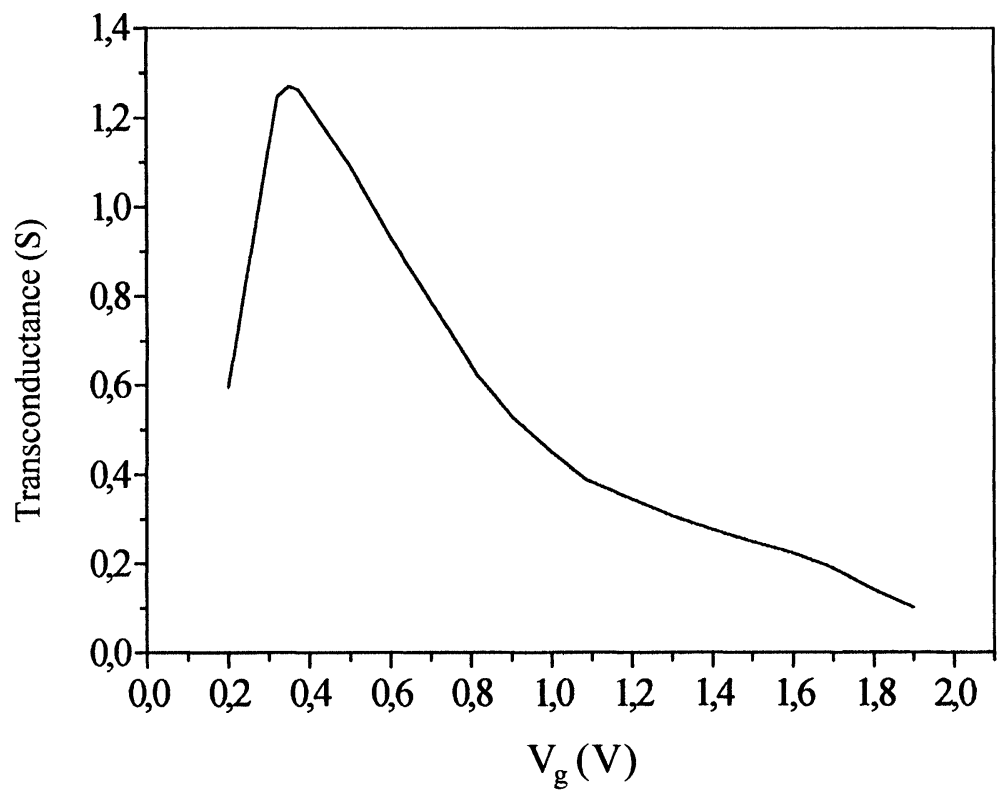

FIGURE 1 Transconductance $g_{m}$ versus the gate voltage of MOSFET with $0.1 \mu \mathrm{m}$ channel length.

Classically, the carrier distribution in a TMOS inversion layer can be described by an exponential function of the effective electric field $F_{\text {eff }}$ to the surface and the space $x[7]$ such as:

$$
n(x)=n_{s} \cdot \exp \left(-\beta \cdot F_{\text {eff }} \cdot x\right)
$$

where $n_{s}$ is the surface carrier concentration and $\beta=q / k T$, with $k T / q$ being the thermal voltage.

The average value of local mobility $\mu(\mathrm{x})$ on all the space is written as such:

$$
\mu_{\mathrm{eff}}=\frac{\int_{0}^{\infty} \mu_{b} n(x) d x}{\int_{0}^{\infty} n(x) d x}
$$

where $\mu_{\mathrm{b}}$ is the bulk silicon mobility in the absence of surface roughness.

However, in our approach of surface roughness scattering states that the mobility $\mu(x)$ vanishes within a layer close to the $\mathrm{Si} / \mathrm{SiO}_{2}$ 
interface, whose thickness $\Delta$ is characteristics of the interface micro roughness [2]. Therefore, $\mu(\mathrm{x})$ can be represented by step function of space, such that one gets from relations (3) and (4):

$$
\begin{aligned}
\mu_{\mathrm{eff}} & =\frac{\int_{\Delta}^{\infty} \mu_{b} n(x) d x}{\int_{0}^{\infty} n(x) d x} \\
& =\mu_{b} \cdot \exp \left(-\beta \cdot \Delta \cdot F_{\mathrm{eff}}\right)
\end{aligned}
$$

This relation (5) shows that, in our surface roughness scattering analysis, the effective mobility is an exponentially decreasing function both of the interface micro roughness amplitude and of the effective electric Field $F_{\text {eff }}$ which is related to the inversion charge $Q_{i}$ and to the depletion charge $Q_{d}$ by:

$$
\mu_{\mathrm{eff}}=\frac{\eta \cdot Q_{i}+Q_{d}}{\varepsilon_{S i}}
$$

where $\varepsilon_{\mathbf{S i}}$ is the silicon permittivity and $\eta$ is weighting factor equal to $1 / 2$ for electron and $1 / 3$ for hole, respectively [5]. In strong inversion, i.e., above the threshold voltage $V_{t}$, the inversion charge $Q_{i}$ is indeed a linear function of gate voltage $\mathrm{V}_{\mathbf{g}}$ :

$$
Q_{i} \cong C_{o x} \cdot\left(V_{g}-V_{t}\right)
$$

Which give to effective mobility $\mu_{\mathrm{eff}}$ the form:

$$
\mu_{\mathrm{eff}}=\mu_{0} \cdot \exp \left\lfloor-\theta \cdot\left(V_{g}-V_{t}\right)\right\rfloor
$$

where $\theta=\beta \cdot \eta \cdot \Delta \mathrm{C}_{\mathrm{ox}} / \varepsilon_{\mathrm{Si}}$ is the mobility attenuation factor and $\mu_{0}=\mu_{\mathrm{b}} \cdot \exp \left(-\beta \cdot \Delta \cdot \mathrm{Q}_{\mathrm{d}} / \varepsilon_{\mathrm{Si}}\right)$ is the low gate voltage mobility.

The mobility $\mu_{0}$ variation with the surface roughness $\Delta$ is in good agreement with Ohmi results [1] (Fig. 2).

The parameters $\theta$ and $\mu_{0}$ have a physical origin, since they are directly related to intrinsic parameters of component. On the other hand, the development of the expression (8) give:

$$
\frac{1}{\mu_{\mathrm{eff}}} \cong \frac{1}{\mu_{0}} \cdot\left[1+\theta \cdot\left(V_{g}-V_{t}\right)+\frac{\theta^{2}}{2} \cdot\left(V_{g}-V_{t}\right)^{2}+\cdots\right]
$$

Which is a generalisation of previous models. Therefore relation (9) provides for the first time, a quantitative relationship between the 


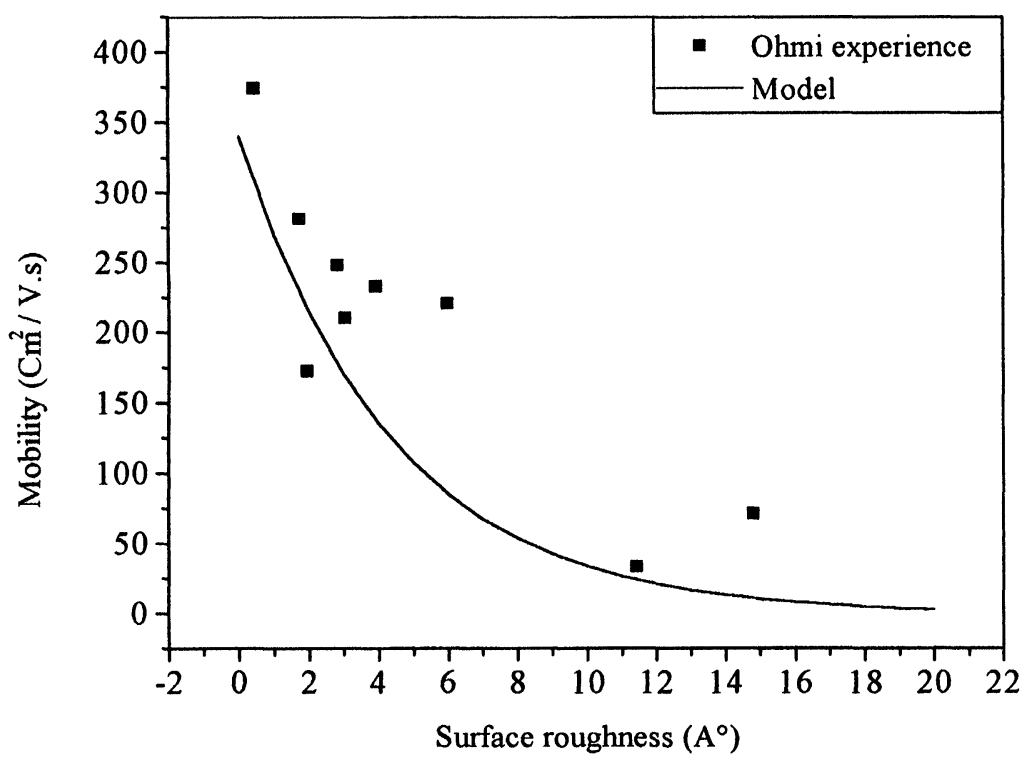

FIGURE 2 Mobility versus surface roughness $\Delta$.

classically measured first order mobility attenuation factor $\theta$ and the surface roughness amplitude $\Delta$.

The previous mobility model has been tested on $\mathrm{N}$ channel transistor. The main parameters were $t_{o x}=5.5 \mathrm{~nm}$, aspect ratio $\mathrm{W} /$ $\mathrm{L}=4 / 0.1$, channel doping in the range $10^{15}-10^{16} / \mathrm{cm}^{3}, \mu_{0}=530 \mathrm{~cm}^{2} /$ $\mathrm{V} \cdot \mathrm{s}$. The effective mobility were calculated as is usual from the ohmic conductance of the device such as, $\mu_{\text {eff }}=L_{d} /\left[W_{o x}\left(V_{g}-V_{t}\right) V_{d}\right]$, with $V_{d}$ being the drain voltage $\left(V_{d}=20 \mathrm{mV}\right)$.

Taking into account the effect of series resistance, the expression $\mu_{\mathrm{eff}}$ becomes:

$$
\mu_{\mathrm{eff}}=\frac{\mu_{0}}{\left[\exp \left[\theta \cdot\left(V_{g}-V_{t}\right)\right]+\frac{K}{V_{d}} \cdot R_{s d} \cdot\left(V_{g}-V_{t}\right)\right]}
$$

with $\mathrm{K}=(\mathrm{W} / \mathrm{L}) \cdot \mu_{0} \cdot \mathrm{C}_{\mathrm{ox}} \cdot \mathrm{V}_{\mathrm{d}}$.

The relation (10) clearly shows that the presence of series resistance modify the behavior of the effective mobility with gate voltage. In all the cases the effective mobility decrease exponentially with the 


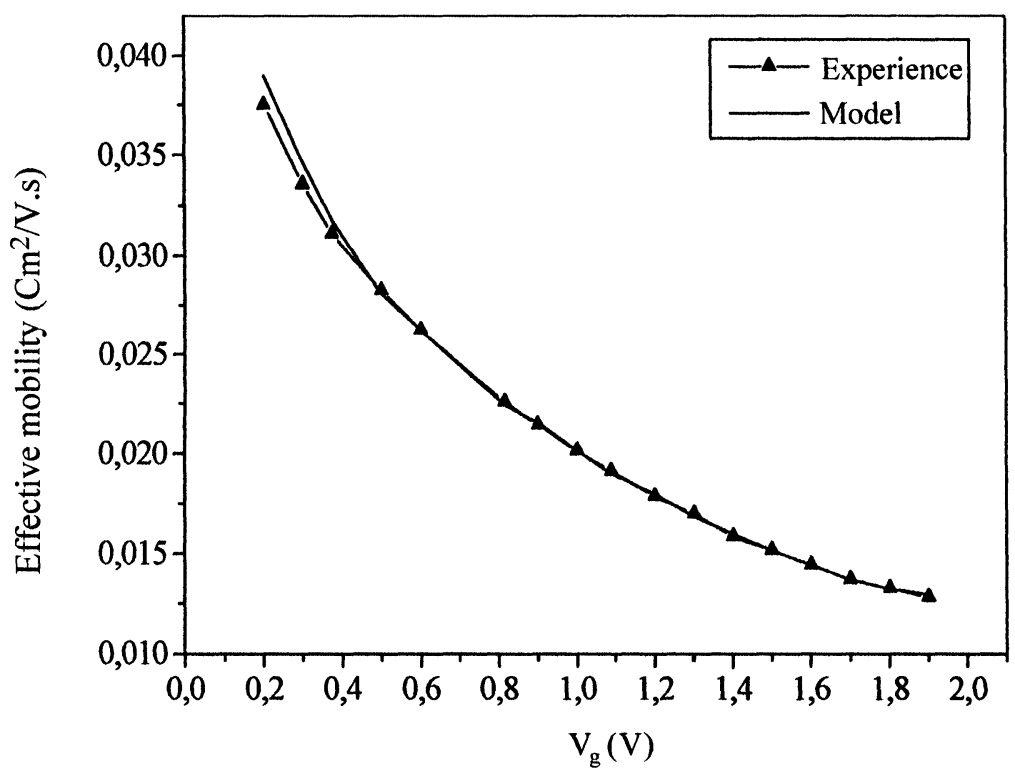

FIGURE 3 Experimental and theoretical effective mobility versus gate voltage $\mathrm{V}_{\mathbf{g}}$.

inversion charge (Fig. 3). The expression drain current function of gate voltage becomes

$$
I_{d}\left(V_{g}\right)=\frac{K}{\left[\exp \left[\theta \cdot\left(V_{g}-V_{t}\right)\right]+\alpha \cdot\left(V_{g}-V_{t}\right)\right]}
$$

with $\alpha=\left(\mathrm{K} / \mathrm{V}_{\mathrm{d}}\right) \cdot \mathbf{R}_{\mathrm{sd}}=(\mathrm{W} / \mathrm{L}) \cdot \mu_{0} \cdot \mathrm{C}_{\mathrm{ox}} \cdot \mathbf{R}_{\mathrm{sd}}$.

\section{RESULTS AND DISCUSSION}

The transfer characteristic $I_{d}$ versus gate voltage $V_{g}$ shows that this result is validated experimentally to many devices with different lengths, the theoretical and experimental curves are in good perfect agreement (Fig. 4). In this letter, an original model especially conceived for the extraction of the series resistance is presented. The exploitation of $Y\left(V_{g}\right)$ characteristic, transfer characteristic $I_{d}\left(V_{g}\right)$ and gate voltage $V_{g}$ variations enables us to determine $R_{s d}$ as function of gate voltage. This implies that the drain current is affected by series 


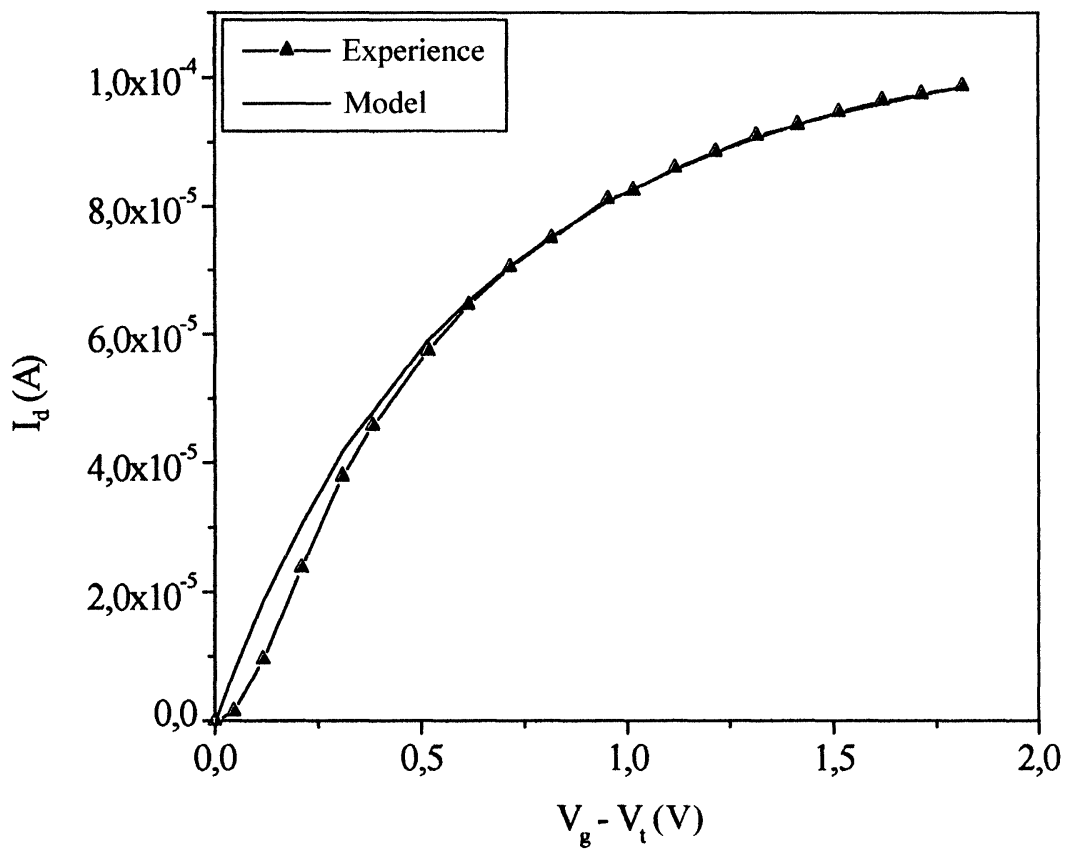

FIGURE 4 Theoretical and experimental drain current $I_{d}$ versus gate voltage $V_{g}$.

resistance $\mathbf{R}_{\text {sd. }}$. Nevertheless, the amplitude of the current, the transconductance, and other quantities are altered by the series resistance effects.

\subsection{Extraction of $V_{t}$ and $\mu_{0}$ Parameters}

In relatively weak field we use the model (1) as an approximation of our model, we plot this function $Y\left(V_{g}\right)=I_{d} / \sqrt{g_{m}}$ where $g_{m}$ represents the transconductance. The parameters $V_{t}$ and $\mu_{0}$ can be extracted from the $y$ axis intercept and slope, respectively, of the observed straight lines of $\mathrm{Y}$ versus $\mathrm{V}_{\mathrm{g}}$ plot (Fig. 5).

\subsection{Determination of Mobility Attenuation Coefficient $\theta$}

For the strong values of $\mathrm{V}_{\mathrm{g}}$, we plot $\log \left(\mu_{\mathrm{eff}}\right)$ which proportional to $\log \left(\mathrm{I}_{\mathrm{d}} /\left(\mathrm{V}_{\mathrm{g}}-\mathrm{V}_{\mathrm{t}}\right)\right)$ versus $\left(\mathrm{V}_{\mathrm{g}}-\mathrm{V}_{\mathrm{t}}\right)$ which is straight line of the slope $\theta$ (Figs. 3 and 4). 


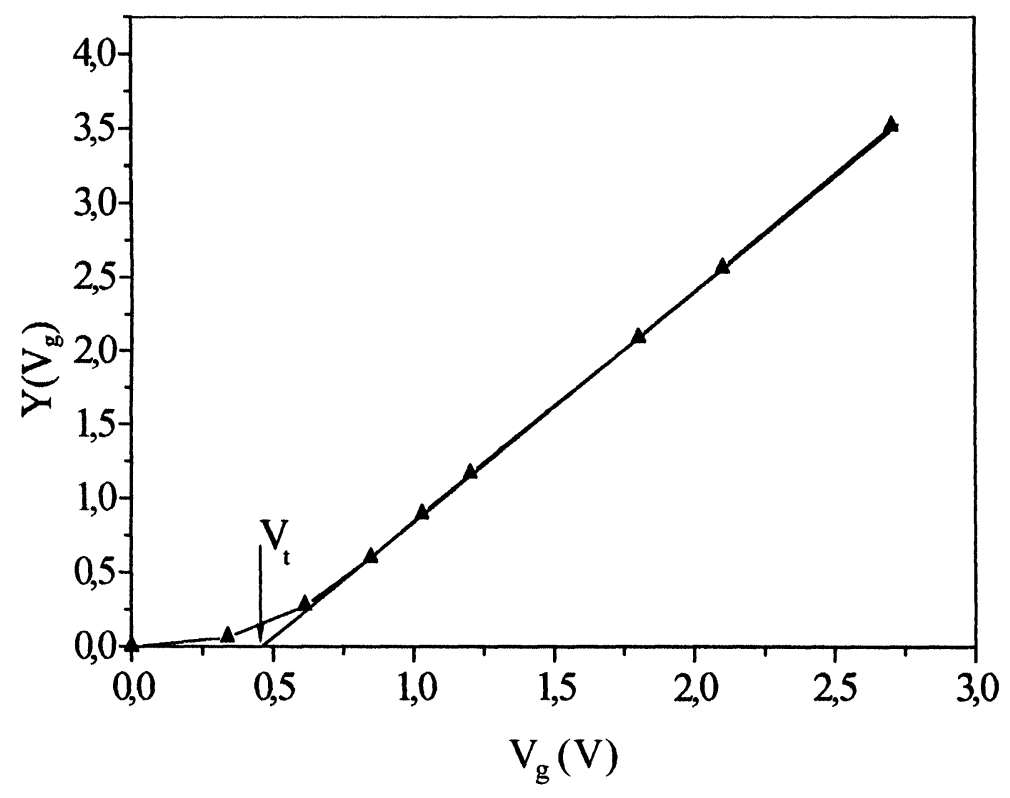

FIGURE 5 Characteristic $\mathrm{Y}\left(\mathrm{V}_{\mathrm{g}}\right)$ of NMOS using the first mobility approach.

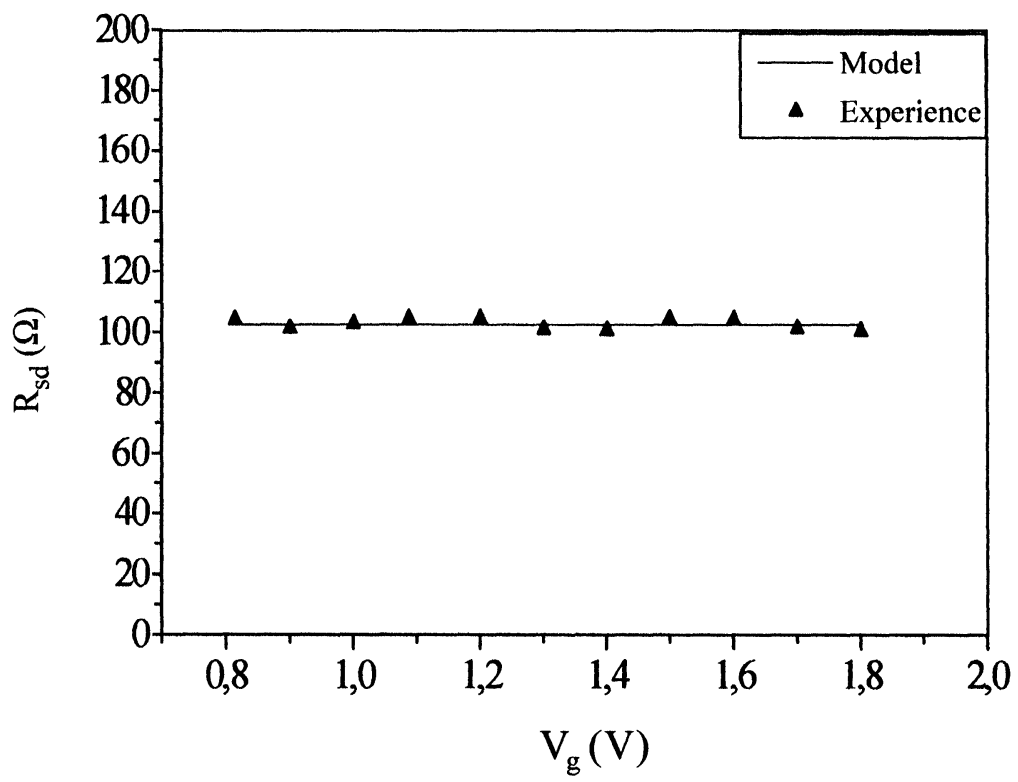

FIGURE 6 Theoretical and experimental characteristics of series resistance versus gate voltage $\mathbf{V}_{\mathbf{g}}$. 


\subsection{Determination of Series Resistance}

Noticing that the expression of series resistance $\mathbf{R}_{\text {sd }}$ is:

$$
R_{s d}=V_{d} \cdot\left[\frac{1}{I_{d}}-\frac{1}{I_{d}^{*}}\right]
$$

Knowing that the relation between $I_{d}$ and $I_{d}^{*}$ according to our model, we write as:

$$
\frac{1}{I_{d}^{*}}=\frac{g_{m}}{I_{d}^{2}} \cdot \frac{\left(V_{g}-V_{t}\right)}{\left[1-\theta \cdot\left(V_{g}-V_{t}\right)\right]}
$$

The $\mathbf{R}_{\text {sd }}$ expression becomes:

$$
R_{s d}=V_{d} \cdot\left[\frac{1}{I_{d}}-Y^{-2} \cdot \frac{\left(V_{g}-V_{t}\right)}{\left[1-\theta \cdot\left(V_{g}-V_{t}\right)\right]}\right]
$$

Plotting this expression versus $\left(\mathrm{V}_{\mathrm{g}}-\mathrm{V}_{\mathrm{t}}\right)$ we obtain the value of $\mathrm{R}_{\mathrm{sd}}$ (Fig. 5).

\section{CONCLUSION}

In this work we have shown a new model of the series resistance in MOSFET device. On the basis of the effective mobility model which considers that the attenuation of mobility with strong inversion is mainly due to the surface roughness scattering. Considering that the mobility is vanishing on the deepness $\Delta$ of some Angstroms and constant in the bulk. We have shown that the effective mobility decreases exponentially in function of the inversion charge; these have permitted us to generalise all the other models used, explain the negative transconductance observed at very strong inversion, to evaluate the different parameters of the model but particularly to determine the value of series resistance.

\section{References}

[1] Ohmi, T., Kotani, K., Teramoto, A. and Myashita, M. (1991). IEEE Trans. Electron. Dev. Letters, EDL12, 652-654.

[2] Ando Fowler and Stem (1987). Rev. Mod. Phys., 54, 448-513. 
[3] Sun, S. C. and Plummer, J. D. (1980). IEEE Trans. Electron. Dev., ED27, $1497-1508$

[4] Ghibaudo, G. and Balestra, F. (1988). Solid State Electroniques, 31, 105-108.

[5] Rais, K., Emrani, A., Balestra, F. and Ghibaudo, G., Electronic Device Operation, Honululu, USA, p. 52, May, 1993.

[6] Rais, K., Ghibaudo, G. and Balestra, F., IEEE Semiconductor Interface Specialist Conference Sisc 93, Fort Lauderdale, USA, Dec., 1993. 

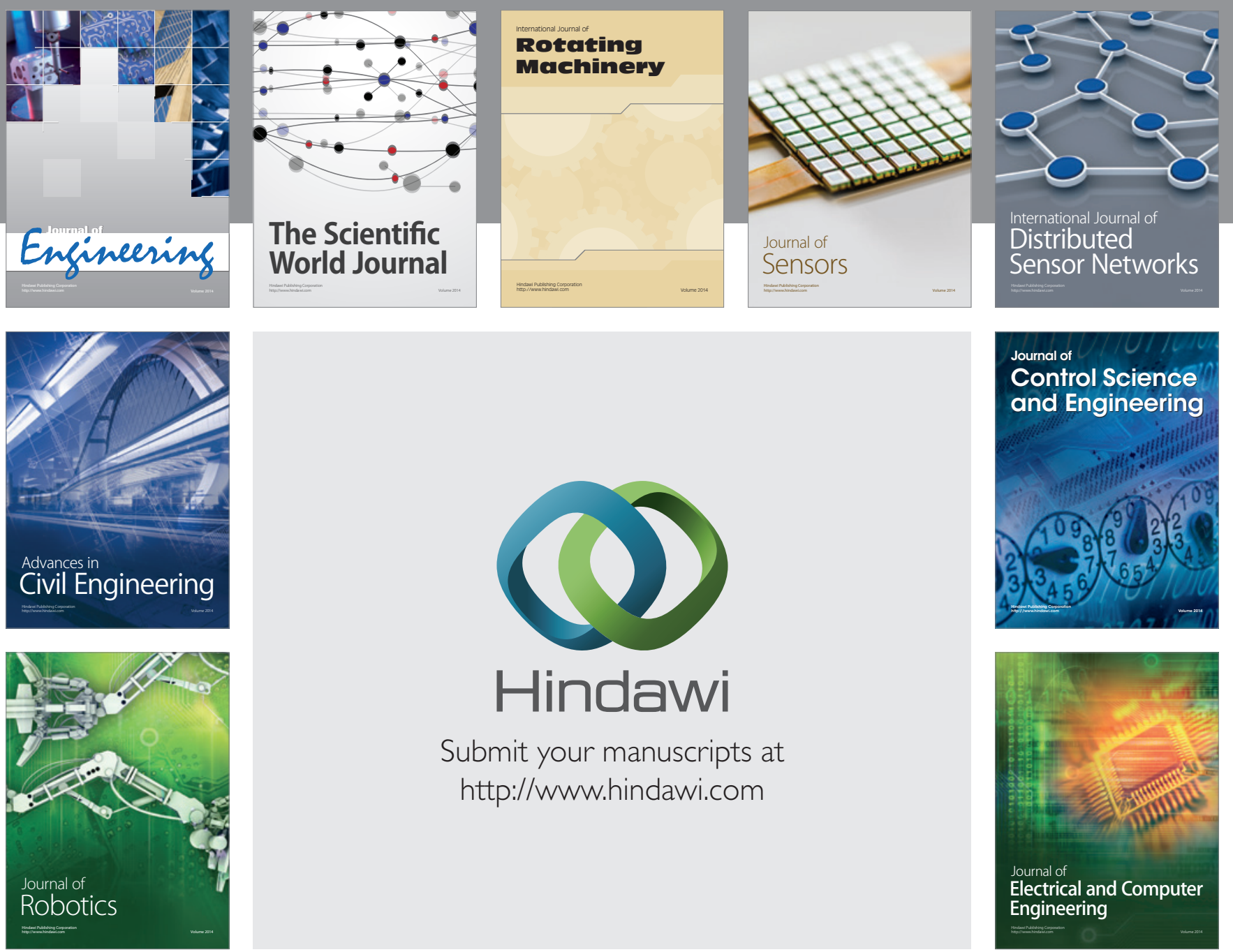

Submit your manuscripts at

http://www.hindawi.com
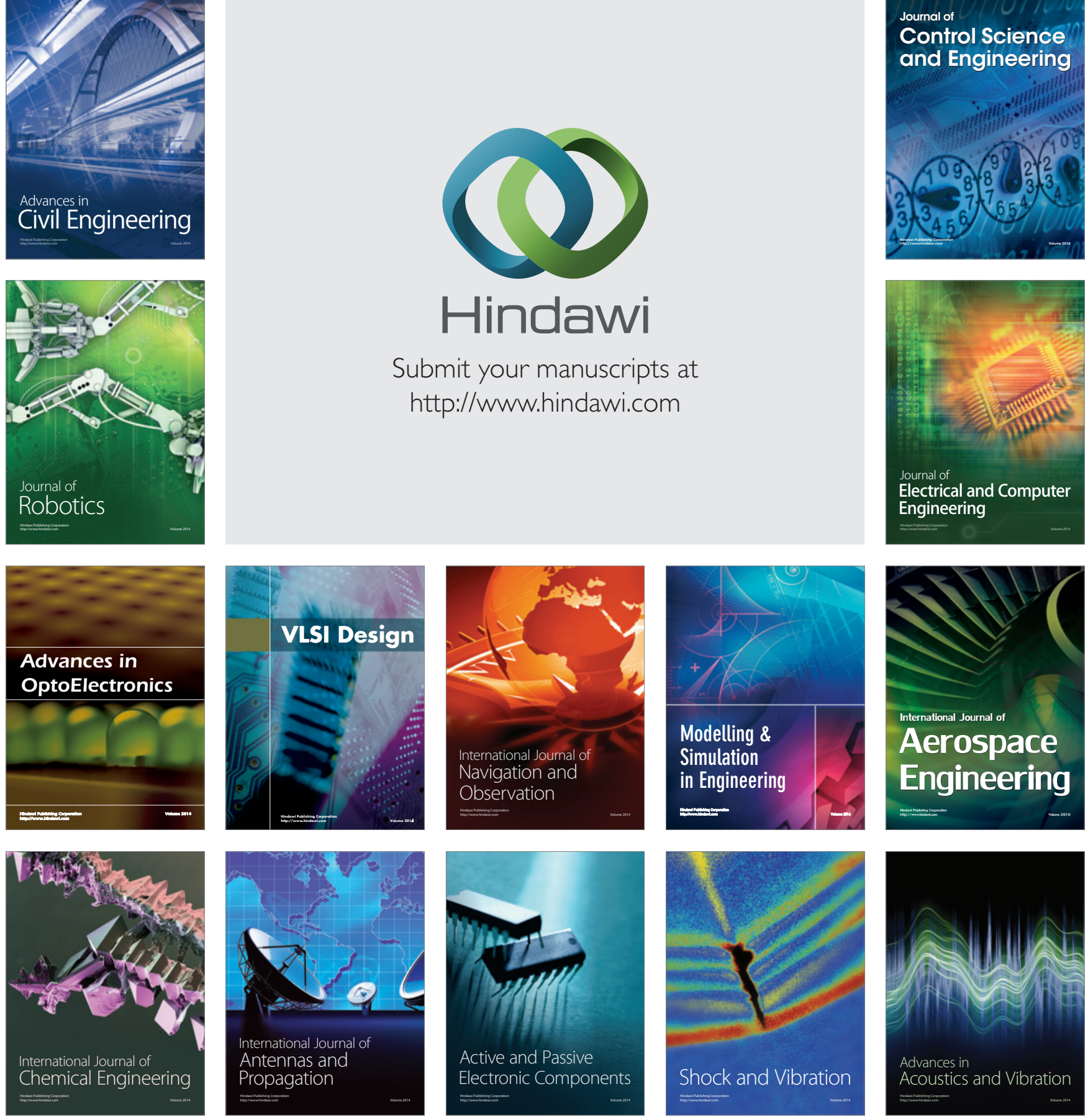